\title{
REGISTRATION AND NORMALIZATION TECHNIQUES FOR ASSESSING BRAIN FUNCTIONAL IMAGES
}

\author{
Y.L. LIAO', N.T. CHIU', C.M. WF.NG ${ }^{3}$, AND Y.N. SUN ${ }^{1}$ \\ 'Department of Computer Science and Information Engineering, \\ ${ }^{2}$ Department of Nuclear Medicine, National Cheng Kung University, \\ ${ }^{3}$ Computer Center, Tainan Woman's College of Art and Science, \\ Tainan, Taiwan
}

\begin{abstract}
Tc-99m HMPAO is a typical tracer used in the analysis of cerebral blood flow. Single photon emission computed tomography (SPECT) brain imaging utilizing Tc-99m HMPAO is thus a popular method to assess brain function. In this paper, we present an image analysis system for SPECT images that performs a series of image processing procedures including $3 D$ image registration, graylevel normalization, and brain extraction, which map all the $3 D$ brain data to the same space for further analysis. Afterward, the aligned images undertake a standard statistical analysis, the paired $t$ test, to detect the areas that have significant deviations between the two images. The statistical t map is then represented with a color plot for each brain slice to indicate the activation foci. The resulting maps are found very helpful to doctors for the lesion evaluation and localization in the clinical diagnosis of schizophrenic patients.
\end{abstract}

Biomed Eng Appl Basis Comm, 2003 (June); 15: 87-94.

\section{INTRODUCTION}

Nowadays, the great progress in neuroimaging has made the nuclear medicine techniques play a more and more important role in the exploration of the relations between human behavior and brain functions. Functional imaging techniques, such as positron emission tomography (PET), single photon emission computed tomography (SPECT), and functional magnctic resonance imaging ( $M R \mathrm{R} I$ ), provide the important information about regional cerebral blood flow ( $\mathrm{rCBF}$ ) or metabolism in the specific brain areas.

Tc-99m HMPAO was developed and later verified

Received: April 21. 2003; Accepted: May 14, 2003

Correspondence: Prof. Y.N. Sun

Department of Computer Science and Information

Engineering, National Cheng Kung University.

Tainan, Taiwan

E-Mail: ynsun@mail.ncku.edu.tw that this tracer can pass through the normal blood-brain barrier into the brain tissue steadily and abidingly. We can thus use this characteristic to relate the blood flow with brain activity using SPECT technology because areas with increased blood flow take up more radiotracer than areas with less blood flow. Therefore, it has been popular to use such methods for the diagnoses of miscellaneous brain diseases, such as schizophrenia, epilepsy, and Alzheimer's disease.

Statistical Parametric Mapping (SPM) is a wellknown softwarc package designed to analyze functional neuroimaging data. SPM99, the latest version, is now widely used to produce a statistically meaningful comparison between groups of images by many researchers and doctors. This software is mainly composed of two steps, spatial pre-processing and statistical analysis, which form a standard procedure in this field.

There are two major issues in the spatial preprocessing stage: image registration and gray-level normalization. Image registration is the process of 
determining the correspondence between all points in two images of the same scene. It has applications in many fields, especially in medical imaging, the one we concern in this paper. Due to the differences in imaging conditions, such as imaging device, imaging duration, or isotope dosage, we are likely to encounter great variations in image quality for most radiological examinations. It has become a popular research topic to overcome these variations and achieve good registration results. Because only when we align two images to the same physical space, we then can perform the subsequent analyses meaningfully.

In the last two decades, many medical image registration methods were developed for various cases [1-2]. Here we are interested is the voxel-based registration methods which operate directly on the image gray-level values, without prior data processing or segmentation. A lot of registration criteria have been proposed for intra-modality and inter-modality registration [3]. Woods et al. [4-5] used the ratio image uniformity to measure the variation of the image intensity of corresponding voxel pairs. Roche et al. [6] took the correlation ratio as a measure under the assumption of the functional dependence between the image intensities. Information-theoretic measures derived from information theory have also attracted many researchers these years because of their satisfactory results. Consequently, mutual information (MI), normalized mutual information (NMI), conditional entropy, and entropy correlation coefficient (ECC) thus become widespread similarity measures [710].

Besides the geometric registration, some image gray-level value manipulations are also needed to make the assessed image sets under the consistent conditions. Although the intensity normalization may dominate the quality of experiments, little investigation has been made to see how the choice of these methods affects the analytic results. In HMPAO imaging, the gray-level values are strongly related to the dosage and radioactivity decay of the isotope, it is believed that there should be a statistical model to relate the two image sets under comparison. Such a model can naturally be applied for the intensity normalization in our experiments. Gullion et al. [11] and Marco et al. [12] compared some methods in their papers and got some interesting conclusions.

After the image registration and intensity normalization, the data are ready for statistical analysis. A voxel by voxel hypothesis testing approach, which reliably identifies regions showing significant experimental effects of interest, is usually applied in this stage. In SPM, a general linear model, including t-test, analysis of variance (ANOVA), analysis of covariance (ANCOVA), linear regression, multiple regression, $F$-test, etc., has to be determined according the properties of image data. After applying the selected model, we can obtain a statistic image, called statistical parametric map. Based on the statistic image, the next step is to reliably locate voxels where effects of interest are exhibited while limiting the false positives. Although there are various methods, including multiple comparisons, random field theory, and spatial levels of inference and power, are developed for the analysis of the statistic image. For simplicity, our system adopts the color-encoding scheme to directly depict the resulting statistic maps.

In our case, the image data belongs to the category of intra-modality (SPECT) and inter-subject. We adopt the maximization of mutual information [8] as the registration criterion, which has the advantage of no user interaction and preprocessing. After the registration, we use a least-squares criterion with linear regression [13] to model the gray-level normalization problem. The paired $t$ test is then chosen to find the areas of great deviation in statistical meaning. The resulting statistic map is then color encoded to illustrate the areas with significant function differences. The detailed description of our methods will be given in the following sections.

\section{MATERIALS AND METHODS}

\subsection{Data Acquisition}

In this study, fifteen schizophrenic patients joined the experiment. In order to compare the brain function for some visual stimulation, two sets of images were acquired in two stages, with stimulation and under rest, for each patient in this experiment. In the first stage, we performed the Wisconsin Card Sorting Task (WCST) [14], which has been used to assess dysfunction of the prefrontal cortex and basal ganglia, to the patient. Then a dose of $15 \mathrm{mCi} \mathrm{Tc}-99 \mathrm{~m}$ HMPAO was injected 2 minutes after the start of WCST. Here, we used a triple-headed rotating gamma camera (Multispect3; Siemens, Hoffman Estates, USA) with ultra high-resolution fan-beam collimators. The SPECT data were acquired over a circular $360^{\circ}$ rotation, 120 steps, 25 seconds per 3 steps, in a $128 \times$ $128 \times 16$ matrix. In the second stage, the patient was under rest condition before being injected with another dose of $25 \mathrm{mCi} \mathrm{Tc}-99 \mathrm{~m}$ HMPAO. The acquisition time was 15 seconds per 3 steps in this stage, and the time interval between two stages of imaging was about 30 minutes. The whole imaging procedure is illustrated in Fig.1. Reconstruction was performed by filtered backprojection using a Butterworth filter with attenuation correction by the Chang method. The data were reformatted to provide one-pixel thick $(2.89 \mathrm{~mm})$ transverse slices parallel to the canthomeatal line. Each 
volume image set may consist of 45 up to 65 transverse slices. Now the raw image data contains a lot of information of the brain function after stimulation and under rest. We will go on to the next step for the detailed image processing procedures.

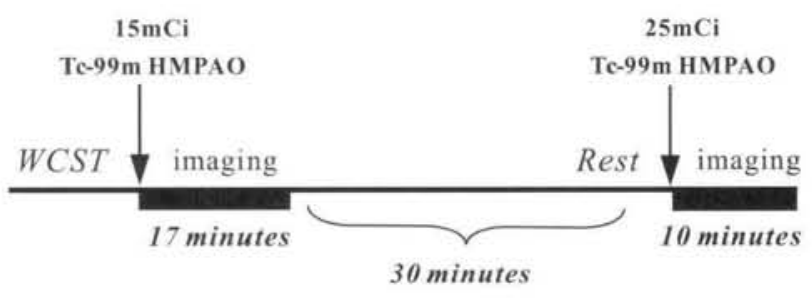

Fig. 1. Brain SPECT imaging procedures.

\subsection{Mutual Information Theory}

Image registration can be viewed as the most important step because only when all volume image data are precisely registered, the brain regions that respond to the visual stimulation can be correctly located. Traditional image registration method usually minimizes the intensity difference of all corresponding voxels between two images [15]. This is under the assumption that two images are the same when they are perfectly aligned. Nevertheless, in inter-modality case or in some special circumstance like ours that all images were acquired under different conditions, we can only say that all images are highly correlated but not the same.

To overcome the problem of intensity inconsistency, we adopt the mutual information as the registration criterion [8]. One intuitive advantage of this method is that we can omit the intensity adjustment step before registration, that is, the raw image data can be directly used to perform the MI registration.

MI is based on the information theory [16]. Define two random variables, $A$ and $B$, with marginal probability distribution, $p_{A}(a)$ and $p_{B}(b)$, and joint probability distribution, $p_{A B}(a, b)$. MI, $I(A, B)$, measures the degree of dependence of $A$ and $B$ by measuring the distance between the joint distribution $p_{A b}(a, b)$ and the distribution associated with the case of complete independence $p(a) \cdot p_{s}(b)$, by means of the relative entropy or the Kullback-Leibler measure [17], i.e.,

$$
I(A, B)=\sum_{a, b} p_{A, B}(a, b) \log \frac{p_{A, B}(a, b)}{p_{A}(a) \cdot p_{B}(b)}
$$

$\mathrm{MI}$ is related to the entropy by the equations

$$
\begin{aligned}
I(A, B) & =H(A)+H(B)-H(A, B) \\
& =H(A)-H(A \mid B) \\
& =H(B)-H(B \mid A)
\end{aligned}
$$

with $\mathrm{H}(A)$ and $\mathrm{H}(B)$ being the entropy of $\mathrm{A}$ and $\mathrm{B}$, respectively, $\mathrm{H}(\mathrm{A}, \mathrm{B})$ their joint entropy, and $\mathrm{H}(\mathrm{A} \mid \mathrm{B})$ and $\mathrm{H}(\mathrm{B} \mid \mathrm{A})$ the conditional entropy of $A$ given $B$ and of $B$ given $A$, respectively. Here set theory representations of the entropies are illustrated in Fig. 2 ., and the definitions are

$$
\begin{gathered}
H(A)=-\sum_{a} p_{A}(a) \log p_{A}(a) \\
H(A, B)=-\sum_{a, b} p_{A, B}(a, b) \log p_{A, B}(a, b) \\
H(A \mid B)=-\sum_{a, b} p_{A, B}(a, b) \log p_{A \mid B}(a \mid b)
\end{gathered}
$$
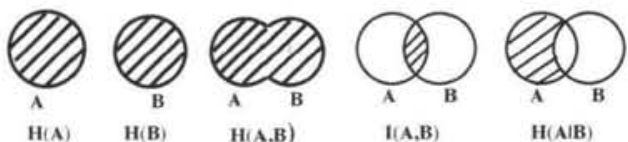

Fig. 2. Set theory representations of the entropies and $\mathrm{MI}$.

\subsection{Joint Histogram}

To apply the MI concept to the image domain, we must give each probability distribution, $p_{A}(a), p_{B}(b)$, and $p_{A B}(a, b)$, an explicit definition. There are two main methods, Parzen window [9] and joint histogram [10], to estimate the probability value. Due to the consideration of computation burden, we choose to build the two-dimensional joint histogram. The joint histogram of an image pair can be defined as a function of two variables, $A$, the gray-level intensity in one image, and $B$, the gray-level intensity in the other image. Each value $h$ at the entry $(A, B)$ is the number of corresponding pairs having gray-level $\mathrm{A}$ in the first image and gray-level $B$ in the second image. Estimation for the joint probability distribution $p_{A B}(a$, $b$ ) are obtained by normalizing the joint histogram of the image pair as

$$
p_{A, B}(a, b)=\frac{h(a, b)}{\sum_{a, b} h(a, b)}
$$

Thus the two marginal probability distributions can also be obtained directly from the joint probability distribution as 


$$
\begin{aligned}
& p_{A}(a)=\sum_{b} p_{A, B}(a, b) \\
& p_{B}(b)=\sum_{a} p_{A, B}(a, b)
\end{aligned}
$$

The MI registration criterion $\mathrm{I}(\alpha)$ is then evaluated from (1) with transformation parameter $\alpha$, and the optimal registration parameter $\alpha^{*}$ is found when $\mathrm{I}(\alpha)$ is maximal as

$$
\alpha^{*}=\arg \max _{\alpha} I(\alpha)
$$

From (1) and (8)-(10), we can find that the values of entries in the joint histogram are the only quantities to calculate the MI value. Therefore, the way we build the joint histogram play a very important role because the quality of the estimated joint histogram has a decisive influence on the accuracy of this method.

Let $\boldsymbol{F}$ denote the transformed image, on which we apply the transformation $\mathrm{T} \alpha$, and the $\boldsymbol{R}$ the reference image. Since the transformed position from $\boldsymbol{F}$ will not always coincide with a grid point of $\boldsymbol{R}$, we may need to introduce an interpolation scheme. Nearest neighbor (NN) interpolation is too rough to guarantee the subvoxel accuracy. Trilinear (TRI) interpolation is the method commonly used for most of the registration criteria, including mutual information. However, this method may introduce new intensity values, which are originally not present in the reference image, and leads to unpredictable changes in the cost function. To overcome this problem, the partial volume (PV) interpolation method was proposed for computing the mutual information [10]. Instead of interpolating new intensity values, the contribution is distributed to all the neighboring voxels to make the joint histogram smoother and the resulting MI is less affected with this artifact. Unfortunately, the other type of artifact may still be introduced with PV interpolation [18]. Some other methods [19][20] were proposed to better cope with this new artifact. The researches to compute MI more precisely and efficiently are still hot issues nowadays.

To take account of the stability of the registration behavior, we choose the TRI interpolation because of its superior consistency compared with the PV interpolation. Consistency is defined here that the forward transformation should be the inverse of the backward transformation (Fig. 3). The total number of bins in our joint histogram is $1024 \times 1024$ because the maximal intensity of all raw data is 590 , and we don't rescale the image intensities to a new range. The binning technique in histogram computation may save quite a lot of computation and is studied in the subsequently research.

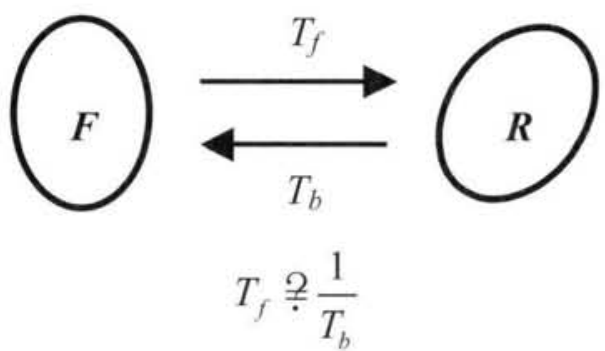

Fig. 3. Registration consistency.

\subsection{Image Registration}

Image registration, brain extraction, and intensity normalization are three main steps of image processing in our system. In image registration, the registration procedures can be divided in two parts, intra-subject and inter-subject ones. Along the registration procedures, each registration step is performed with two volume images at a time.

In the intra-subject part, we use the images, which were acquired at rest, as the template images. The images acquired under visual stimulation are thus registered with respect to the template images. We can therefore obtain fifteen sets of transformation parameters, where each set is for a specific patient. In the inter-subject part, we select an image acquired at rest, out of the fifteen patients, as the standard template. All the other fourteen images have to be registered to the selected one. Here we obtain fourteen sets of transformation parameters. Thus we obtain totally twenty-nine sets of transformation parameters, fifteen ones for intra-subject and fourteen for intersubject. They are then utilized to transform all images to the same physical space. For the images at rest, they are directly transformed with the fourteen sets of parameters to the standard template. And for the images under stimulation, they have to undertake the intra-subject transformation and then the inter-subject transformation. A simple registration diagram is given in Fig. 4, where each arrow indicates that one image register to another one.

For simplicity, the transformations in the intrasubject part have been restricted to the rigid body transformations with three translation parameters and three rotation parameters. And under the consideration of the different brain sizes between different patients, we add the scaling parameters in the inter-subject part. The images are initially positioned such that their geometric centers coincide together. Then the Powell's method [21] is used to search the optimal transformation parameter $\alpha$ to maximize the mutual information $I(\alpha)$. 


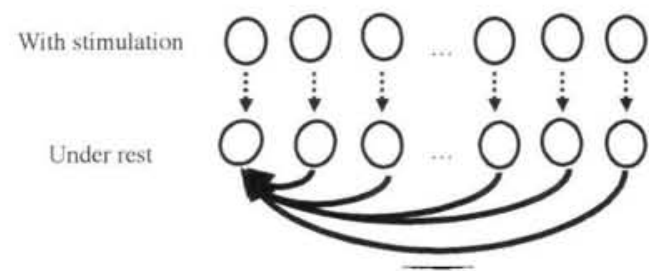

Fig. 4. Registration scheme. Intra-subject registration is performed first (dotted arrows), and then inter-subject one (solid arrows).

\subsection{Brain Extraction}

Following the registration, we also need to adjust the image intensity. Obviously, only the brain area should join the calculation of parameter adjustment, so we have to separate the brain from the skull, muscle, and scalp. Since the SPECT image is a low-resolution image, which lacks of the anatomical information, we cannot define the brain contour as precisely as the MR image. We utilize the optimal thresholding method to extract the brain region [22]. This algorithm assumes that regions to segment have two major lobes in the gray-level distribution and uses an iterative threshold selection scheme to obtain the optimal threshold. The threshold value can be mostly obtained in four to ten iterations. This method is quite simple but gives good segmentation results and greatly reduces the possibility of inconsistency in extracting brain regions by hand.

\subsection{Intensity Normalization}

As a result of different imaging conditions, the image intensities obtained from each scan cannot be compared directly. Just like the spatial registration, we also adopt an intensity normalization process along with each step of spatial registration for both the intraand inter-subject parts to transform the gray-level values of the acquired images to a consistent base that is the intensity distribution of the standard template image. For each pair of images to register, the relationship between intensities of the two images can be described by a linear model with the equation $\mathrm{y}=\alpha$ $+\beta \mathrm{x}$, where $\alpha$ is the additive parameter, $\beta$ is the scaling parameter, and $\mathrm{x}$ and $\mathrm{y}$ are the corresponding voxel values in the two images [13]. We use a leastsquares criterion with the linear regression method to evaluate the two parameters. They are calculated immediately after each geometric registration step. After the adjustment according to the model, the wellregistered and well-normalized data are valid for the subsequent statistical analysis.

\subsection{Statistical Analysis}

In this experiment, we want to detect the area with significant difference under two statuses, which are with visual stimulation and at rest. The paired $t$ test is thus suitable to these types of paired data, where two images are for each patient. At first, we will get a statistic t map, with each voxel a test statistic value, in the following way. The data consists of 15 independent image pairs $\left(\mathrm{x}_{1 j}, \mathrm{y}_{1 j}\right),\left(\mathrm{x}_{2 j}, \mathrm{y}_{2 j}\right), \ldots,\left(\mathrm{x}_{15 j}, \mathrm{y}_{15 j}\right)$, with $j$ denotes the position in the image. Let $d_{1 j}=x_{1 j}-y_{1 j}$, $\mathrm{d}_{2 j}=\mathrm{x}_{2 j}-\mathrm{y}_{2 j}, \ldots, \mathrm{d}_{15 j}=\mathrm{x}_{15 j}-\mathrm{y}_{15 j}$, so the $\mathrm{d}_{i j}$ 's are the differences within pairs. Then the test statistic value at each voxel position is

$$
t_{j}=\frac{\bar{d}_{j}}{s_{j} / \sqrt{n}},
$$

where $d_{j}$ and $s_{j}$ are the sample mean and standard deviation of the $d_{i j}$ 's with $\mathrm{n}=15$.

Now we have a statistical volume data, with each voxel representing its paired t-test value. A color lookup table of our previous work [23] is then used to create a color plot for the statistic $t$ map. By using the color mapping technique, red and blue colors indicate whether the region contains relatively more cerebral flow or not. The green areas are thus where significant difference is not present. The distribution in the resulting color map can thus reveal a complete picture of functional variations between the set of images at rest and the ones under stimulation from the statistical point of view. Visualization of the statistic value for each image slice will help doctors on locating the regions with positive and negative responses quickly.

\section{RESULTS}

We adopt a volume rendering technique called semi-boundary rendering [24] to reconstruct the volume data. It can be used to evaluate the registration performance visually. In order to compare the relative position for data before and after registration, we used the image blending technique to see how our method works. We use the dark red color to stand for the transformed volume, and the reference volume is colored with dark green. The overlapping parts thus become in white color and shaded with respect to its surface normal. Fig. 5(a) and 5(b) are the data before and after registration, respectively, in the intra-subject registration stage. Fig. 6(a) and 6(b) are those in the inter-subject registration stage. We can obviously find that the results are pretty good visually.

Tables I and II show the average and maximum error of the registration consistency. Each time we use a pair of images to perform the experiment and get a set of transformation parameters $\boldsymbol{T}$. Then the roles of the transformed and reference images are exchanged to get another set of transformation parameters $T^{\prime}$. If our registration is robust and consistent, $\boldsymbol{T}+\boldsymbol{T}^{\prime}$ will get 


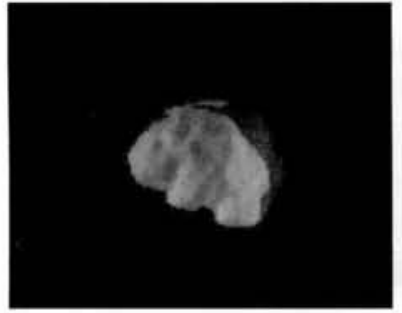

(a)

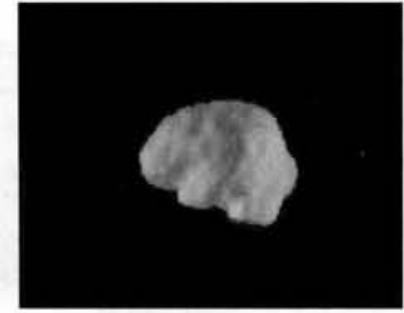

(b)
Fig. 5. Intra-subject registration. The translation parameters are $0.314,6.864,-4.470$ in $x-, y-, z-$ direction (in voxels), and the rotation parameters are $-14.539,-1.752,-0.592$ about $x-, y-, z$-axis (in degrees) in this case. (a) Before registration. (b) After registration.

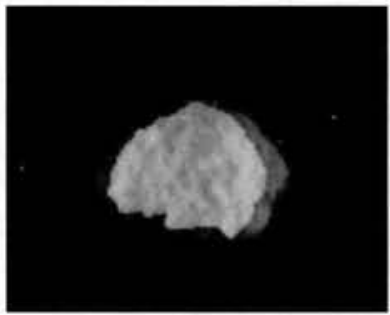

(a)

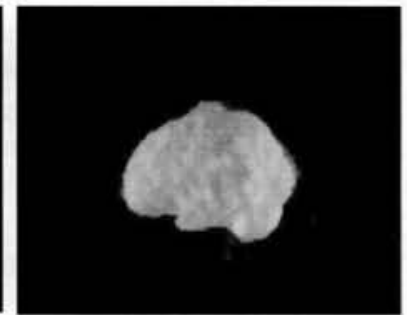

(b)
Fig. 6. Inter-subject registration. The translation parameters are 1.445, 5.618, 0.919 in $x-, y-, z-$ direction (in voxels), and the rotation parameters are $1.915,2.716,1.782$ about $x-, y-, z$-axis (in degrees) in this case. (a) Before registration. (b) After registration.

zero values for each parameter. Not surprisingly, the errors of inter-subject are larger than those of intrasubject. This is mainly because in two out of fifteen cases the rotation parameters are trapped in the local minima and get larger deviations in the inter-subject registration. However, we can still guarantee the subvoxel accuracy according the average error.

We also present a pair of image slice before and after intensity normalization to verify the normalization performance (see Fig. 7). Actually, it is difficult to claim that our gray-level normalization is indeed the most suitable method for our case. However, the subsequent statistical analytic results show great consistence with the physiological expectation. Therefore, the proposed image-processing procedure has revealed its good applicability in our experiments.

Finally, a color plot of each slice, which shows the paired $t$-test result, is presented in Fig. 8. The red areas stand for the positive result that the brain is more activated under visual stimulation than at rest. And the blue areas are thus the contrary results where more blood flows for the at-rest case. We can find that the red areas concentrate in the hindbrain from the $25^{\text {th }}$ to $35^{\text {th }}$ slice (the total number of slices in the $t$ map volume image is 61 ). In brain anatomy, these area indeed correspond to the primary visual reception area. Therefore, this experiment successfully conforms to our expectation.

Table I. Intra-subject registration consistency error ( $T_{x}, T_{y}, T_{z}$ in voxels and $R_{x}, R_{y}, R_{z}$ in degrees).

\begin{tabular}{|l|c|c|c|c|c|c|}
\hline & $\mathbf{T}_{\mathbf{x}}$ & $\mathbf{T}_{\mathbf{y}}$ & $\mathbf{T}_{\mathbf{z}}$ & $\mathbf{R}_{\mathbf{x}}$ & $\mathbf{R}_{\mathbf{y}}$ & $\mathbf{R}_{\mathbf{z}}$ \\
\hline Average & 0.033 & 0.061 & 0.107 & 0.359 & 0.234 & 0.303 \\
\hline Maximum & 0.098 & 0.214 & 0.328 & 2.118 & 0.692 & 0.721 \\
\hline
\end{tabular}

Table II. Inter-subject registration consistency error ( $T_{x}, T_{y}, T_{z}$ in voxels and $R_{x}, R_{y}, R_{z}$ in degrees).

\begin{tabular}{|l|c|c|c|c|c|c||}
\hline & $\mathbf{T}_{\mathbf{x}}$ & $\mathbf{T}_{\mathbf{y}}$ & $\mathbf{T}_{\mathbf{z}}$ & $\mathbf{R x}_{\mathbf{x}}$ & $\mathbf{R}_{\mathbf{y}}$ & $\mathbf{R}_{\mathbf{z}}$ \\
\hline Average & 0.188 & 0.235 & 0.173 & 3.516 & 1.225 & 0.515 \\
\hline Maximum & 0.882 & 1.548 & 0.613 & 15.778 & 4.664 & 1.854 \\
\hline
\end{tabular}

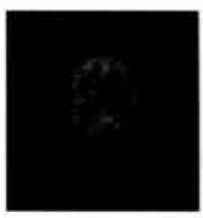

(a)

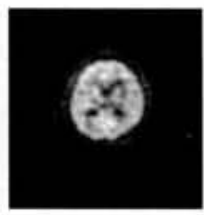

(b)

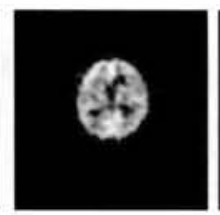

(c)

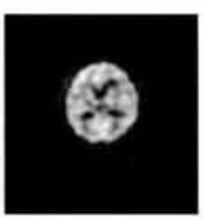

(d)
Fig. 7. Gray-level normalization. The $33^{\text {rd }}$ slice of a patient's volume image. (a) Image acquired with stimulation before normalization. (b) Image acquired at rest before normalization. (c) Image in (a) after normalization. (d) Image in (b) after normalization.

\section{DISCUSSION AND CONCLUSION}

In this paper, we have developed an integrated image analysis system for the evaluation of functional variations between the Tc-99m HMPAO brain SPECT images. The proposed system consists of the procedures for 3D image registration, gray-level normalization, brain extraction, and statistical analysis. The procedures for registration and normalization are designed for the brain SPECT images, which can be subsequently analyzed under a unique basis. The 


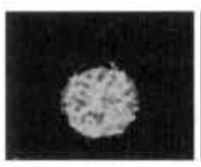

(a)

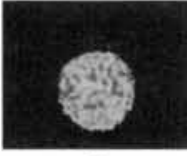

(b)

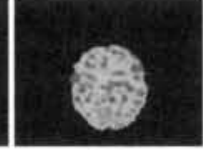

(c)

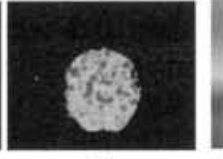

(d)
Fig. 8. Slices of statistical $t$ map image. (a) - (d) The $20^{\text {th }}, 25^{\text {th }}, 30^{\text {th }}, 35^{\text {th }}$ slice, respectively. (e) The color bar used to map the statistic value into the color plot.

discrepancies between the two sets of SPECT images are then assessed by a standard statistical analysis procedure. The results not only show great reliability in image registration but reveal that the functional assessment is effective and consistent with our clinical expectation.

To define the brain region for image registration is usually a difficult task in many image registration methods. We adopt the mutual information (MI) for registration and use the whole brain data, and thus the segmentation step is avoided. Here, we utilize the trilinear interpolation in the MI computation. It achieves accurate results for the experimental image sets.

The proposed method is a useful tool that provides good capability in the comparative evaluation and visualization for SPECT images. Because of its good adaptability in image registration and normalization, it can also be adapted for other ncuroimaging cases where data are usually acquired under inconsistent imaging conditions. In the future study, we will cmploy the multi-resolution technique to overcome the local optimization problem and to further reduce the computation burden. Histogram binning will be used together. Naturally, a better interpolation method will also be considered in the registration process.

\section{REFERENCES}

1. J.V. Hajnal, D.L.G. Hill, and D.J. Hawkes, Medical Image Registration, CRC Press, 2001.

2. J.B.A. Maintz and M.A. Viergever, "A survey of medical image registration", Med. Image Anal., Vol. 2, Iss. 1, 1998, pp. 1-36.

3. J. West, J.M. Fitzpatrick, et al., "Comparison and evaluation of retrospective intermodality brain image registration techniques", J. Comput. Assist. Tomogr., Vol. 21, Iss. 4, 1997, pp. 554-566.

4. R.P. Woods, J.C. Mazziotta, and S.R. Cherry, "Rapid Atomated Agorithm for Aigning and Rslicing PET Images", J. Comput. Assist. Tomogr., Vol. 16, Iss. 4, 1992, pp. 620-633.
5. R.P. Woods, J.C. Mazziotta, and S.R. Cherry, "MRI-PET Registration with Automated Algorithm", J. Comput. Assist. Tomogr., Vol. 17, Iss. 4, 1993, pp. 536-546.

6. A. Roche, G. Malandain, X. Pennec, and N. Ayache, "The correlation ratio as a new similarity measure for multimodal image registration", in Proc. of First Int. Conf. on Medical Image Computing and Computer-Assisted Intervention (MICCAI'98), Vol. 1496 of Lecture Notes in Computer Science, 1998, Cambridge, U.K., pp. 1115-1124, Springer Verlag.

7. A. Collignon, F. Maes, D. Delaere, D. Vandermeulen, P. Suetens, and G. Marchal, "Automated multi-modality image registration based on information theory", in Information Process. Med. Imaging 1995, Y. Bizais, C. Barillot, and R. Di Paola, Eds, Kluwer Academic, Dordrecht, The Netherlands, 1995, pp. 263-274.

8. F. Maes, A. Collignon, D. Vandermeulen, G. Marchal, and P. Suetens, "Multimodality Image Registration by Maximization of Mutual Information", IEEE Trans. Med. Imag., Vol. 16, no. 2, 1997, pp. 187-198.

9. W.M. Wells III, P. Viola, H. Atsumi, S. Nakajima, and R. Kikinis, "Multi-modal volume registration by maximization of mutual information", Med. Image. Anal., Vol. 1, no. 1, 1996, pp. 35-51.

10. C. Studholme, D.L.G. Hill, and D.J. Hawkes, "An overlap invariant entropy measure of 3D medical image alignment", Pattern Recognit., Vol. 32, Iss. 1, 1999, pp. 71-86.

11. C.M. Gullion, M.D. Devous, Sr., and A.J. Rush, "Effects of four normalizing methods on data analytic results in functional brain imaging", Biol. Psychiatry, Vol. 40, no. 11, 1996, pp. 1106-1121.

12. R.G. Marco, E.J.A. Garica-Iturrospe, L.F. Lopez, M.R.C. Mendez, O.H. Rodriguez, A.D. Ramirez, J.H. Martinez, and M.S. Keshavan, "Hypofrontality in schizophrenia: influence of normalization methods", Prog. Neuro-Psychopharmacol \& Biol. Psychiatry, Vol. 21, no. 8, 1997, pp. 1239-1256.

13. C. Perault, D. Papathanassiou, H. Wampach, P. Vera, A. Kaminska, C. Chiron, P. Peruzzi, and J-C, Liehn, "Computer-aided intrapatient comparison of brain SPECT images: the gray-level normalization issue applied to children with epilepsy", J. Nucl. Med., Vol. 43, no. 6, 2002, pp. 715-724.

14. O. Monchi, M. Petrides, V. Petre, K. Worsley, and A. Dagher, "Wisconsin Card Sorting Revisited: Distinct Neural Circuits Participating in Different Stages of the Task Identified by Event-Related Functional Magnetic Resonance Imaging", J. Neurosci., Vol. 21, no. 19, 2001, pp. 7733-7741. 
15. P. Thevenaz, U.E. Ruttimann, and M. Unser, "A Pyramid Approach to Subpixel Registration Based on Intensity", IEEE Trans. Image Processing, Vol. 7, no. 1, 1998, pp. 27-41.

16. T.M. Cover and J.A. Thomas, Elements of Information Theory, John Wiley \& Sons, New York, 1991.

17. I. Vajda, Theory of Statistical Inference and Information, Kluwer Academic, Dordrecht, The Netherlands, 1989.

18. J.P.W. Pluim, J.B.A. Maintz, and M.A. Viergever, "Interpolation artefacts in mutual informationbased image registration", Computer Vision and Image Understanding, Vol. 77, Iss. 2, 2000, pp. 211-232.

19. D. Sarrut and F. Feschet. "The Partial Intensity Difference Interpolation", in International Conference on Imaging Science, Systems and Technology, H. R. Arabnia, eds, CSREA Press, Las Vegas, USA, 1999, pp. 46-51.
20. H.M. Chen and P.K, Varshney, "Registration of multimodal brain images: some experimental results", in Proc. SPIE Conference on Sensor Fusion: Architectures, Algorithms, and Applications VI, Vol. 4731, B.V. Dasarathr, Eds, 2002, pp.122-133.

21. W. H. Press, B.P. Flannery, S.A. Teukolsky, and W.T. Vetterling, Numerical Recipes in C, 2nd ed., Cambridge University Press, Cambridge, U.K., 1992.

22. M. Sonka, V. Hlavac, and R. Boyle, Image Processing Analysis, and Machine Vision, 2nd ed., PWS Publishing, 1999.

23. Y.N. Sun, S.C. Huang, N.T. Chiu, C.Y. Yu, and F.J. Chen, "Bullseye display of cerebral cortical blood flow", IEEE Eng. in Med. and Biol., Vol. 21, Iss. 4, 2002, pp. 79-85.

24. T.Y. Lee, T.L. Weng, and Y.N. Sun, "Optimized Semi-Boundary (SB) Rendering Scheme", J. Inf. Sci. Eng., Vol. 15, no. 6, 1999, pp. 845-858. 